\title{
Nivel de expresión escrita en la carta en alumnos de primaria rural de Tenosique Tabasco
}

\author{
Lázaro Jiménez Ara \\ jimenez752010@hotmail.com \\ Centro Internacional del Posgrado A.C. \\ Escuela Primaria Prof. Florencio Jiménez García \\ https://orcid.org/0000-0002-8753-6929 \\ Mariana de Jesús Centeno Loarca \\ marianacenteno914@hotmail.com \\ Escuela Primaria Valentín Gómez Farías \\ https://orcid.org/0000-0003-4375-5262
}

Martha Isela Baños Dorantes

martha_isela80@outlook.com

Colegio de Bachilleres de Tabasco, Plantel 13

https://orcid.org/0000-0001-5372-8642

\section{RESUMEN}

Nicolás González Cortés

Universidad Juárez Autónoma de Tabasco,

Campus Tenosique, Tabasco, México

https://orcid.org/0000-0001-7336-4524

nicolas.gonzalez@ujat.mx

El objetivo fue explorar el nivel de escritura, usando como recurso la carta, en alumnos de sexto grado de la escuela primaria rural "Valentín Gómez Farías" en el municipio de Tenosique, Tabasco, México. La investigación fue de tipo descriptivo y se diseñó un instrumento de medición de 10 criterios con tres niveles de valorización; alto, medio y deficiente. Se trabajó con un grupo de 30 alumnos: 11 niñas y 19 niños, de 12.3 años de edad.

El tema central del texto en la carta fue la descripción "Mi familia y Yo". Los datos fueron analizados con estadística descriptiva, medidas de tendencia central y de dispersión. Los resultados indicaron que los alumnos presentan un bajo nivel de escritura. Solo el 53.3\% de las niñas alcanzaron un nivel medio, 9.2 puntos porcentuales arriba de los niños (44.1\%), y el $47.2 \%$ de niñas y el 58.6\% de los niños presentaron un nivel deficiente. Se concluye que las niñas tienen mejor nivel de escritura en comparación con los niños, principalmente en los indicadores de vocabulario y ortografía.

Palabras claves: la carta; redacción de textos; educación primaria. 


\title{
Writing level in children of sixth grade of rural primary school in Tenosique Tabasco
}

\begin{abstract}
The objective was to explore the writing level, using the letter as a resource, in sixth grade students from the rural primary school "Valentín Gómez Farías" in the municipality of Tenosique, Tabasco, Mexico. The research was descriptive and a measuring instrument of 10 criteria with three levels of valuation was designed; high, medium and poor. We worked with a group of 30 students: 11 girls and 19 boys, 12.3 years old. The central theme of the text in the letter was the description "My family and I". The data were analyzed with descriptive statistics, measures of central tendency and dispersion. The results indicated that the students present a low level of writing. Only $53.3 \%$ of the girls reached a medium level, 9.2 percentage points above the boys (44.1\%), and $47.2 \%$ of the girls and $58.6 \%$ of the boys presented a deficient level. It is concluded that girls have a better level of writing compared to boys, mainly in vocabulary and spelling indicators.
\end{abstract}

Keywords: the letter; writing texts; primary education.

Artículo recibido: 25enero 2021 Aceptado para publicación: 28febrero2021 Correspondencia: nicolas.gonzalez@ujat.mx Conflictos de Interés: Ninguna que declarar 


\section{INTRODUCCIÓN}

La capacidad de escribir textos descriptivos y argumentativos está generalmente conectada con la capacidad cognitiva, experiencia personal y social. Por ello, se debe construir un conocimiento basado en experiencias y el contraste de ideas, las cuales colaboran mutuamente en la producción de textos de acuerdo a las técnicas desarrolladas por Freinet (Freinet, 1972). La carta o correspondencia escolar es un medio de comunicación interpersonal que propicia pensamientos, sentimientos, ideas, deseos, sueños, confesiones, propósitos, creatividad, cooperación. Este recurso ayuda a los docentes a conocer más afondo a sus alumnos, construyendo relaciones en el grupo, basadas en la cooperación, sin perder de vista la potenciación de la propia individualidad. Así se fomenta un acto de verdadera lectura y escritura; una lectura que va más allá de la mera interacción entre el lector y la información visual que el texto aporta. Son muchos y diversos los valores y apoyos emocionales que suscita la correspondencia: la tolerancia, la sinceridad, la reacción positiva a la frustración, la generosidad, el compartir, la cooperación, la sensibilidad, la capacidad de asombro, la empatía, etc. (SEP, 2011). Al respecto Cassany (2006) indica que la carta es un escrito que obliga al individuo a "dominar muchas habilidades: discriminar las informaciones relevantes, de las irrelevantes estructurarlas en un orden cronológico, comprensible, escoger las palabras adecuadas, conectar las frases entre sí, aplicar las reglas fonéticas y ortográficas, morfosintácticas y léxicas" que permite crear textos con las reglas de adecuación coherencia y cohesión. En México, la educación primaria es un nivel básico de seis grados para niñas y niños de edad promedio de 6 a 12 años. En cada uno de los seis grados de estudios está vigente la lectura y la escritura de textos. Sin embargo, según la Organismo para la Cooperación del Desarrollo Económico (OCDE), en los últimos años en México, el desempeño se encuentra por debajo del promedio en ciencias (416 puntos), lectura (423 puntos) y matemáticas (408 puntos); en estas tres áreas, menos del 1\% de los estudiantes en México logran alcanzar niveles en competencia de excelencia (SEP, 2011). Por lo que Bauté, (2004) define que la correspondencia es un medio para medir y fomentar el lenguaje escrito. Por tanto, el objetivo de este estudio fue medir el nivel de expresión escrita usando como recurso la carta, tomando como tema central "Mi familia y Yo", en niños de sexto grado de primaria de Tenosique, Tabasco, México. 


\section{MATERIALES Y MÉTODOS}

\section{Descripción de la zona de estudio}

El municipio de Tenosique se localiza en la región de los Ríos, al sur del estado de Tabasco, entre los paralelos $17^{\circ} 28.5^{\prime}$ de latitud norte y los $91^{\circ} 25.6^{\prime}$ de longitud oeste, y a $20 \mathrm{msnm}$, colindando al sur con el país de Guatemala. El clima es cálido húmedo con abundantes lluvias todo el año, y una temperatura media anual de $30.5^{\circ} \mathrm{C}$. Las actividades económicas más importantes son el comercio, la agricultura, la ganadería y la pesca. El municipio cuenta con abundantes recursos hídricos, fauna y flora, y un Área Natural Protegida de Flora y Fauna del Cañón del Usumacinta, y entre sus atractivos turísticos está el carnaval más raro del mundo la danza del "Pochó".

\section{Sector educativo en Tenosique}

El municipio cuenta con todos los niveles educativos, desde preescolar hasta dos instituciones de educación superior. En cuanto a nivel Primaria, el municipio cuenta con 83 escuelas de nivel primaria, distribuidas de la siguiente manera: 19 escuelas en cabecera municipal y las demás en la zona rural.

\section{Escuela primaria rural "Valentín Gómez Farías"}

La escuela primaria rural "Valentín Gómez Farías" con clave C.C.T: 27DPR097M zona escolar no. 90 y sector escolar no. 16 y cuenta con una matrícula de 105 alumnos, de los cuales 51(48.6\%) son niñas y $54(51.4 \%)$ son niños. El grupo de sexto grado está integrado por 30 alumnos; $11(36.7 \%)$ son niñas y $19(63.3 \%)$ niños, con una edad promedio de 12.3 años. Los matriculados son atendidos por cuatro maestros frente a grupo, un intendente y un director. La escuela cuenta con cinco salones, servicios sanitarios para niñas y niños, una plazuela de usos múltiples, una cocina de desayunos escolares, la casa del maestro y un salón para la dirección. En la Tabla 1 se presentan los datos de los alumnos de 6to.

Tabla 1. Alumnos de sexto grado de la escuela primaria rural "Valentín Gómez Farías" del municipio de Tenosique Tabasco, México.

\begin{tabular}{llclll}
\hline Zona & Escuela & $\begin{array}{c}\text { No. } \\
\text { alumnos }\end{array}$ & Sexo & $\%$ & Total \\
\hline \multirow{2}{*}{ Rural } & \multirow{2}{*}{\begin{tabular}{l} 
Valentín Gómez Farías \\
\cline { 2 - 5 }
\end{tabular}} & 11 & Niñas & 36.7 & 30 \\
\cline { 3 - 5 } & 19 & Niños & 63.3 &
\end{tabular}

Ubicación geográfica de la escuela primaria rural "Valentín Gómez Farías" 
La escuela "Valentín Gómez Farías" fue fundada en 1983 y se localiza en el ejido Álvaro Obregón, enclavado en la zona sierra de Tenosique y a $30 \mathrm{~km}$ en carretera y terracería de la cabecera municipal. Esta escuela está inmersa en un contexto bilingüe sus habitantes hablan la lengua tzeltal y el español, y con una riqueza de recursos naturales de flora y fauna impresionante. Las actividades económicas que rodean la comunidad, predominan la agricultura, la ganadería y el empleo temporal. En el 2015 se obtuvo el acceso a internet, aunque la disposición de computadoras y uso de celulares es muy limitado entre los alumnos. En la Figura 1 se ubica la localización geográfica de la escuela rural "Valentín Gómez Farías".

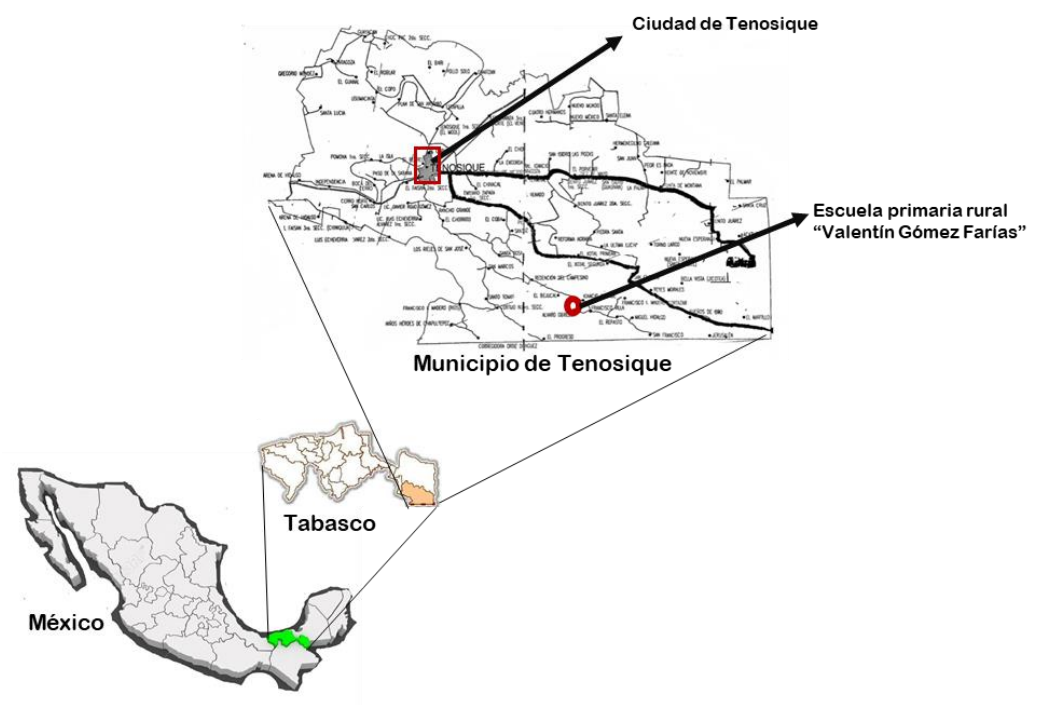

Figura 1. Localización geográfica de la escuela primaria rural "Valentín Gómez Farías" del municipio de Tenosique Tabasco, México.

\section{Tipo de investigación e instrumento de medición}

La investigación fue de carácter descriptivo con variables ordinales y discretas y tipo transversal (Hernández, 2010). Para medir el nivel de escritura en la carta se diseñó una rúbrica, adaptada de Fernández et al. (2019), con 10 criterios, cada uno con tres niveles de valorización: alto, medio y deficiente. El tema a describir en la carta fue "Mi familia y Yo" que consistió primero en leer la descripción de "Mi familia y Yo" del profesor, además se elaboró un cartel para analizar y describir los miembros de una familia típica tabasqueña, integrada por los papás, hermanos, abuelos, tíos y primos, incluyendo hasta las mascotas. Posteriormente a cada uno de los alumnos se le dio una hoja en blanco tamaño carta que contenía tres líneas para que escribieran el nombre de su escuela, su nombre (nombre y apellidos), género y los datos del destinatario (alumnos de sexto grado 
de otra escuela primaria que se les asigno de manera aleatorio); así mismo contenía una pequeña instrucción que indicaba "Describe cómo está integrada tu familia y las actividades que realizan juntos" además se les indico que tenían 20 minutos para realizar la actividad. En la Tabla 2 se muestra la rúbrica de evaluación del nivel de escritura en la carta.

Tabla 2. Nivel de escritura en la carta en niños de sexto grado de primaria.

\begin{tabular}{|c|c|c|c|}
\hline \multirow{2}{*}{ Criterios } & \multicolumn{3}{|c|}{ Nivel de desempeño } \\
\hline & Alto & Medio & Deficiente \\
\hline Idea principal & $\begin{array}{l}\text { Se entiende muy bien la } \\
\text { idea principal. }\end{array}$ & $\begin{array}{l}\text { Contiene parcialmente la } \\
\text { idea principal }\end{array}$ & No contiene la idea principal \\
\hline Oraciones & $\begin{array}{l}\text { Las oraciones están bien } \\
\text { estructuradas y } \\
\text { entienden bien }\end{array}$ & $\begin{array}{l}\text { Algunas oraciones están } \\
\text { mal construidas }\end{array}$ & $\begin{array}{l}\text { La mayoría de las oraciones } \\
\text { no se entienden porque están } \\
\text { mal construidas }\end{array}$ \\
\hline $\begin{array}{l}\text { Uso de signos } \\
\text { ortográficos }\end{array}$ & $\begin{array}{l}\text { En un texto de } 100 \\
\text { palabras usa más } \\
\text { cuatro signos }\end{array}$ & $\begin{array}{l}\text { En un texto de } 100 \\
\text { palabras usa menos de } \\
\text { tres signos }\end{array}$ & $\begin{array}{l}\text { No contiene signos de } \\
\text { puntuación }\end{array}$ \\
\hline Presentación & $\begin{array}{l}\text { Contenido limpio y sin } \\
\text { borrones }\end{array}$ & algunos & Con borron \\
\hline Contenido & $\begin{array}{l}\text { El mensaje en la carta es } \\
\text { coherente y apropiado con } \\
\text { el tema de interés }\end{array}$ & $\begin{array}{l}\text { El mensaje le falta } \\
\text { claridad respecto al tema } \\
\text { de interés }\end{array}$ & $\begin{array}{l}\text { El mensaje no tiene } \\
\text { coherencia respecto al tema } \\
\text { a tratar }\end{array}$ \\
\hline $\begin{array}{l}\text { Organización } \\
\text { espacial }\end{array}$ & $\begin{array}{l}\text { El espacio está } \\
\text { correctamente utilizado y } \\
\text { adecuado para cada una } \\
\text { de las partes de la carta }\end{array}$ & $\begin{array}{l}\text { Es adecuado por lo menos } \\
\text { para dos de las partes }\end{array}$ & $\begin{array}{l}\text { El espacio es adecuado para } \\
\text { al menos una de las partes de } \\
\text { la carta }\end{array}$ \\
\hline Vocabulario & $\begin{array}{l}\text { Se utiliza un vocabulario } \\
\text { rico, con algunas palabras } \\
\text { poco conocidas }\end{array}$ & $\begin{array}{l}\text { Las palabras son } \\
\text { correctas, pero se repiten } \\
\text { mucho. }\end{array}$ & $\begin{array}{l}\text { Vocabulario limitado, } \\
\text { repetitivo y algunas palabras } \\
\text { no son las más adecuadas } \\
\text { para lo que se quiere decir. }\end{array}$ \\
\hline Ortografía & $\begin{array}{l}\text { La carta no contiene } \\
\text { errores de ortografía }\end{array}$ & $\begin{array}{l}\text { Contiene no más de tres } \\
\text { errores de ortografía en } \\
100 \text { palabra }\end{array}$ & $\begin{array}{l}\text { Contiene más de tres errores } \\
\text { de ortografía en } 100 \text { palabras }\end{array}$ \\
\hline Caligrafía & La letra es clara y legible & Letra poco legible & Nada legible \\
\hline $\begin{array}{l}\text { Extensión del } \\
\text { texto (número de } \\
\text { palabras) }\end{array}$ & $\begin{array}{l}\text { Contiene más de } 200 \\
\text { palabras }\end{array}$ & $\begin{array}{l}\text { Contiene entre } 101 \text { a } 199 \\
\text { palabras en el texto }\end{array}$ & $\begin{array}{llll}\begin{array}{l}\text { Contiene menos de } \\
\text { palabras }\end{array} & 100 \\
\end{array}$ \\
\hline
\end{tabular}

\section{RESULTADOS Y DISCUSIÓN}

El 67\% y $33 \%$ de la las niñas obtuvieron un de nivel medio y deficiente, respectivamente en cuanto a la claridad de la idea principal, en comparación con los niños donde el $50 \%$ obtuvo un nivel medio y los demás con nivel deficiente. En el criterio de la estructura de las oraciones, el $41.5 \%$ de las niñas presentaron el nivel alto, en comparación con los 
niños con el 50\%, sobre saliendo en este indicador los niños en la estructura de la oración con sujeto, verbo y predicado. En cuanto al uso de signos ortográficos; uso de coma, punto y coma, dos puntos, paréntesis, signos de admiración, de interrogación, puntos suspensivos. En el criterio sobre la presentación (sin borrones ni rayones) los niños presentaron $44.5 \%$ de nivel medio en relación al 33.3\% que obtuvieron las niñas; los niños presentaron su carta con menos borrones o rayones. En el criterio del contenido de la carta el 66\% de las niñas en comparación con los niños con un 33\% están en el nivel medio, y el $67 \%$ de los niños tienen una deficiencia en este indicador. En la organización espacial de la carta las niñas presentan $58.4 \%$ con relación a los niños con un 50\%. En el uso de vocabulario de igual forma el 50\% de las niñas tuvieron un nivel medio con relación al $33.4 \%$ de los niños. Los alumnos de la escuela rural presentan mayores dificultades en su redacción debido a la lengua materna (tzeltal) ya que como hablan, así escriben. En ortografía el $67 \%$ de los niños tienen problemas de ortografía con relación. En caligrafía se puede observar que los niños dejan claro el desinterés por realizar los trazos adecuados en las letras de sus escritos. En extensión del tanto las niñas y niños tuvieron más de 101 palabras en su carta. En la Tabla 3 se presentan los resultados de nivel de escritura en la carta en niños de sexto grado de escuelas primarias rural.

Tabla 3. Resultados del nivel de escritura en la carta en niños de sexto grado de primarias rural de Tenosique Tabasco, México.

\begin{tabular}{|c|c|c|c|c|c|c|c|c|}
\hline \multirow{2}{*}{ Criterio } & \multicolumn{9}{|c|}{ Población } \\
\cline { 2 - 9 } & \multicolumn{3}{|c|}{11 Niñas $(36.7 \%)$} & \multicolumn{3}{|c|}{19 Niños $(63.3 \%)$} \\
\cline { 2 - 9 } & Alto & Medio & Defic. & \multicolumn{1}{|c|}{$\begin{array}{c}\text { Total } \\
(\mathbf{\%})\end{array}$} & Alto & Medio & Defic. & $\begin{array}{c}\text { Total } \\
(\%)\end{array}$ \\
\hline Idea principal & 0 & 66.7 & 33 & 100 & 0 & 50 & 50 & 100 \\
\hline Oraciones & 0 & 41.7 & 58 & 100 & 0 & 50 & 50 & 100 \\
\hline Uso de signos ortográficos & 8.4 & 33.3 & 58 & 100 & 0 & 27.8 & 72 & 100 \\
\hline Presentación & 8.4 & 33.3 & 58 & 100 & 5.5 & 44.5 & 50 & 100 \\
\hline Contenido & 0 & 66.6 & 33 & 100 & 0 & 33.4 & 67 & 100 \\
\hline Organización espacial & 0 & 58.4 & 42 & 100 & 0 & 50 & 50 & 100 \\
\hline Vocabulario & 0 & 50 & 50 & 100 & 0 & 33.4 & 67 & 100 \\
\hline Ortografía & 0 & 50 & 50 & 100 & 0 & 33.4 & 67 & 100 \\
\hline Caligrafía & 8.4 & 50 & 42 & 100 & 0 & 44.4 & 56 & 100 \\
\hline Extensión de texto & 0 & 72.7 & 27.3 & 100 & 0 & 73.7 & 26.3 & 100 \\
\hline Total & $\mathbf{2 5 . 2}$ & $\mathbf{5 2 3}$ & $\mathbf{4 5 2}$ & $\mathbf{1 0 0 0}$ & $\mathbf{5 . 5}$ & $\mathbf{4 4 0 . 6}$ & $\mathbf{5 5 5 . 3}$ & $\mathbf{1 0 0 0}$ \\
\hline Promedio & $\mathbf{2 . 5}$ & $\mathbf{5 2 . 3}$ & $\mathbf{4 5 . 2}$ & & $\mathbf{0 . 5 5}$ & $\mathbf{4 4}$ & $\mathbf{5 5 . 5}$ & \\
\hline D.E & 4.5 & 13.8 & 11.4 & & 1.9 & 13.3 & 13.5 & \\
\hline
\end{tabular}

*Desviación estándar 
Los docentes actuales nos enfrentamos cambios y medios tecnológicos, debido a ello, los estudiantes tienen intereses que obedecen a esquemas diversos en el mundo digital que han limitado sus capacidades para redactar. Por lo tanto, la escuela moderna exige un docente dinámico que canalice estas realidades para que el alumno encuentre sentido a lo que realiza en la escuela. La siguiente investigación relaciona y reorganiza saberes a fin de proporcionar una manera más (eficiente) de potencializar las habilidades lectoras y al mismo tiempo elevar la calidad de la comunicación escrita en los educandos, a través de la "correspondencia interescolar", condición didáctica que genera enriquecimiento en situaciones comunicativas precisas con destinatarios reales. Esta estrategia propicia en los alumnos, compartir sentimientos, ideas, emociones y conocimiento con sus pares, de una manera emocionante, atractiva y divertida. Genera también una relación estrecha entre el redactor y el receptor e involucra a todos los agentes educativos (madres, padres, alumnos, docentes y directivos). Desarrolla habilidades socio comunicativas. Promueve una comunicación coherente, ordenada y eficiente, y al mismo tiempo estimula la curiosidad por leer. Se pretende fortalecer los lazos de confianza y comunicación entre el docente como guía y el alumno; también aportara dinamismo, responsabilidad y una nueva perspectiva docente.

Los resultados en las evaluaciones diagnósticas, el sentir de los profesores respecto al tema de lectura y escritura, nos preocupa y nos mantiene en alerta, en las diversas reuniones de consejo técnico escolar, coincidimos con maestros que están interesados en trabajar para mejorar y desarrollar la habilidad de lectoescritura como una estrategia de comunicación. Con esta investigación medimos el nivel de expresión escrita de los alumnos, evaluar la parte cognitiva y la contextual, sus escritos en las cartas nos dan pautas para reconstruir nuevas estrategias de aprendizaje en la escritura y en la lectura.

Después de haber realizado el análisis de los resultados de la evaluación, donde se observa que hay un alto porcentaje de deficiencia en la redacción de textos, esto ha sido una constante cada ciclo escolar en los diversos grupos que se han atendido.

Es importante fortalecer las actividades que se realicen cada día con los alumnos, ya que las alumnas del medio rural necesitan una mejor atención en cuanto al lenguaje escrito, específicamente en la redacción.

Arellano (2006) aplicó la técnica de la correspondencia interescolar, ayuda a conocer la realidad de los niños, y la reflexión de lo producido. La capacidad de producir textos está 
generalmente conectada a su experiencia personal y social, luego difunden sus propias creaciones, los cuales genera interés por producir textos de manera descriptiva y argumentativa. Las técnicas Freinet, permitirán la mejora de la escritura y creatividad, también podrán desarrollar un pensamiento crítico y analítico a medida que producen, además conocerán distintas realidades que son compartidas con sus compañeros. Fernández, Montero y Lucero (2019) el objetivo de su investigación fue construir y validar una rúbrica para evaluar relatos (RER) redactados por estudiantes de entre 9 y 14 años, para ello, se analizaron un total de 292 textos producidos por estudiantes escolarizados en estos niveles educativos. Los datos aportados apoyan la validez de la RER, lo que sugiere que tanto la estructura dimensional como la operativización de los criterios y niveles de logro son relevantes y suficientemente discriminativos para su aplicación en estos niveles educativos. Se observado que la mayoría de los profesores expresaron algún tipo de interpretación crítica desarrollando un pensamiento complejo de argumentación y análisis histórico. La variable edad no arrojó resultados destacables, pero sí se observaron diferencias respecto a la variable territorial, predominando la visión negativa-crítica en regiones donde se desarrollan movimientos independentistas. En otros trabajos adaptan la batería de los procesos de escritura PROESC evalúa cada uno de los aspectos que constituyen el sistema de escritura, desde los más complejos, como la planificación de las ideas, hasta los más simples, como es la conversión fonema - grafema en sílabas, han demostrado tener confiabilidad y validez, habiendo corroborado el modelo teórico de los procesos de escritura, bajo el enfoque cognitivo. Por otra parte, se evalúa la confiabilidad y validez de la medición de evaluación de escritura (WAM), desarrollada para reflejar las habilidades que se espera que los niños de diferentes habilidades logren en la expresión escrita, también se encontraron diferencias significativas entre niños de diferentes edades y habilidades de escritura. Los resultados indican que el instrumento tiene una utilidad potencial para los profesionales que evalúan la escritura de los niños. Fernández (2017) mostró que la coevaluación, apoyada en una rúbrica, desencadenó una mejora significativa en la organización y el contenido del texto; mientras que el alumnado que fue evaluado por el profesor mejoró significativamente en los aspectos gramaticales y, sobre todo, ortográficos. Finalmente, se discuten las implicaciones de los nuevos conocimientos y recursos generados en las prácticas evaluativas de la competencia escritora que se llevan a cabo en nuestro sistema educativo. Por su parte Graham y Perin 
(2007) indican que existe una preocupación considerable de que la mayoría de los adolescentes no desarrollen la competencia por escrito que necesitan para tener éxito en la escuela, el lugar de trabajo o sus vidas personales. Una explicación común de por qué los jóvenes no escriben bien es que las escuelas no hacen un buen trabajo al enseñar esta compleja habilidad. Los autores realizaron un metanálisis de la literatura de intervención de escritura (grados 4-12), enfocando sus esfuerzos en estudios experimentales y cuasi experimentales. Jonsson y Svingby (2007) presentaron en sus resultados el potencial para la autorregulación y la autoeficacia de los estudiantes, la calidad de la evaluación y la mejora de la enseñanza. Pero es necesario desarrollar rubricas que cumplan con los criterios de fiabilidad y validez. La fiabilidad de la rúbrica se ha evaluado a través de la consistencia interna de lo que se utilizó Alfa de Cronbach. Se obtuvieron coeficientes de confiabilidad considerados aceptables, pero pueden mejorarse. Salvador (2000) aplicó un método psicolingüístico, derivado de la "gramática de la narración", al análisis del corpus (72 textos escritos), estableciendo diversas categorías textuales en el nivel macroestructural. El análisis permitió establecer las estructuras narrativas textuales, características de los alumnos del segundo ciclo de Educación Primaria. El factor diferencial más significativo resultó ser el rendimiento académico de los alumnos, frente a otros, como la edad, el género o la clase social. Yan et al. (2012) indican que el conocimiento del vocabulario, la habilidad de dictado, la edad, el género y la conciencia fonológica incluidos en una ecuación de regresión, se explicó el 35\% de la variación en la calidad de escritura de los 9 años. Con las variables de velocidad de procesamiento, nomenclatura acelerada y fluidez de escritura a mano incluidas adicionalmente como un bloque, se explicó una variación adicional del 12\% en la ecuación.

\section{CONCLUSIÓN}

En esta investigación se usó la carta como recurso para medir el nivel de escritura en alumnos de sexto grado de primaria del medio rural. La mayoría de los alumnos no lograron obtener un nivel alto de redacción, solo el 2.52 de las niñas y el 0.55 de los niños lograron obtener este nivel. La mayoría de los alumnos obtuvieron un nivel medio, las niñas presentaron un 53.3\%, 9.2 puntos porcentuales por arriba de los niños con un $44.1 \%$, mientras que el 45.2 de las niñas y el 55.5\% de los niños obtuvieron un nivel deficiente. Las niñas sobresalen por arriba de 10 puntos porcentuales en los indicadores en la idea 
principal, contenido, vocabulario y ortografía, mientras que los niños únicamente sobresalieron en la estructura de las oraciones y una presentación sin borrones ni rayones.

\section{LISTA DE REFERENCIAS}

Arellano, L. (2006). Técnica Freinet: Aplicación del texto libre en la escuela primaria. Universidad pedagógica nacional, México, 1-60. http://200.23.113.51/pdf/22916.pdf

Baute, C. E. (2004). Las correspondencias escolares. México: biblioteca de escuela moderna.

Cassany, D. (2006). Describir el escribí. Como se aprende a escribir. Barcelona: Paidos.

Fernández, M. (2017). Co-evaluación de textos narrativos en la educación obligatoria. Universidad de Extremadura.

Fernández, M., Lucero, M., Montanero, M. (2016). Rojo sobre negro. ¿Cómo evalúan los maestros las redacciones de sus estudiantes? Revista de educación, 63-82.

Fernández, M., Montanero, M., \& Lucero, M. (2019). La evaluación de la competencia narrativa en la educación básica. Revista de la educación, 85-112.

Freinet, C. (1972). Los métodos naturales III: el aprendizaje de la escritura. Fontanella y Laia.

Graham, S., \& Perin, D. (2007). A meta-analysis of writing instruction for adolescent students. Journal of Educational Psychology.

Jonsson, A., \& Svingby, G. (2007). The use of scoring rubrics: reliability validity and educational consequences. Educational Research Review, 130-144.

Salvador, F. (2000). Habilidades narrativas de alumnos de Educación Primaria en la producción de textos escritos. Enseñanza, 17-18, 145-163.

SEP 2011, S. (2011). Mapa curricular de la educación básica. MÉXICO.

Yan, C., McBride-Chang, C., Wagner, R., Zang, J., Wong, A., \& Shu, H. (2012). Writing quality in Chinese children: speed and fluency matter. Reading and Writing. 